\title{
Comparison of Erythrocyte Traits Among European, Japanese and Korean
}

\author{
Ji-sun Kwon and Sangsoo Kim* \\ Department of Bioinformatics and Life Science, Soong- \\ sil University, Seoul 156-743, Korea
}

\begin{abstract}
Erythrocyte traits are heritable and indirect indicators of blood diseases caused by erythrocyte, but their genetic factors are largely unknown. So we performed genome-wide association study in 8,842 Korean individuals to identify genetic factors influencing erythrocyte traits. We identified 40 associations for three erythrocyte traits at genome-wide significance levels $\left(p<1 \times 10^{-6}\right)$. We compared these associated loci with those reported in genome-wide association studies of European and Japanese. Our findings include previously identified loci (HBS1L-MYB, TMPRSS6, USP49 and CCND3) in other studies and novel associations (MRDS1/OFCC1, CSDE1, NRAS and 8 other loci). For example, SNP rs4895440 of HBS1L-MYB intergenic region on chromosome 6q23.3 is one of the most associations influencing erythrocyte traits $\left(p=8.33 \times 10^{-27}\right)$.
\end{abstract}

Keywords: erythrocyte, GWAS, hematocrit, hemoglobin, red blood cell

\section{Introduction}

Erythrocytes (Red blood cells) such important cells as a function of delivering Oxygen $\left(\mathrm{O}_{2}\right)$ to the body tissues are the most common type of blood cell. The count and volume of the erythrocytes in blood are high heritable and it is different between individuals. Erythrocyte traits include the hemoglobin $(\mathrm{Hb})$, hematocrit $(\mathrm{Hct})$, red blood cell (RBC) which are commonly used indicators in the clinic. Blood diseases caused by erythrocyte are anemias, hemolysis, polycythemias. These are widely associated with other diseases such as cardiovascular diseases, hypertension, but genetic factors of erythrocyte traits are poorly proved.

Genome-wide association studies (GWAS) identify a number of loci associated with common diseases with

${ }^{*}$ Corresponding author: E-mail sskimb@ssu.ac.kr Tel +82-2-828-7190, Fax +82-2-820-0816 Accepted 29 August 2010 quantitative traits. We analyzed GWA scanning data of erythrocyte traits for 8,842 Korean individuals (Fig. 1). To date, samples of several studies associated with erythrocyte traits are European origin, Japanese, and Chinese, and it remains unclear whether the same loci affect erythrocyte traits in Korean. Hence we compared genetic variations that affected erythrocyte traits among European, Japanese and Korean (Fig. 2).

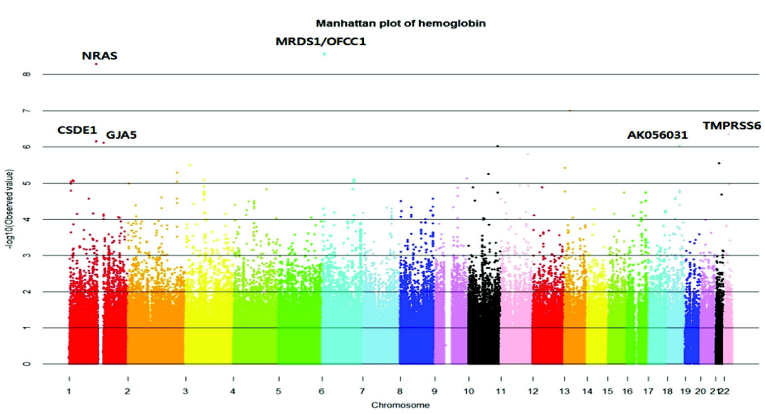

(a)
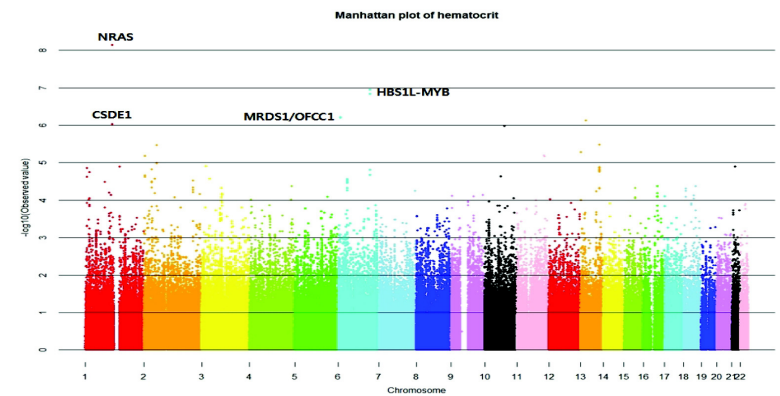

(b)

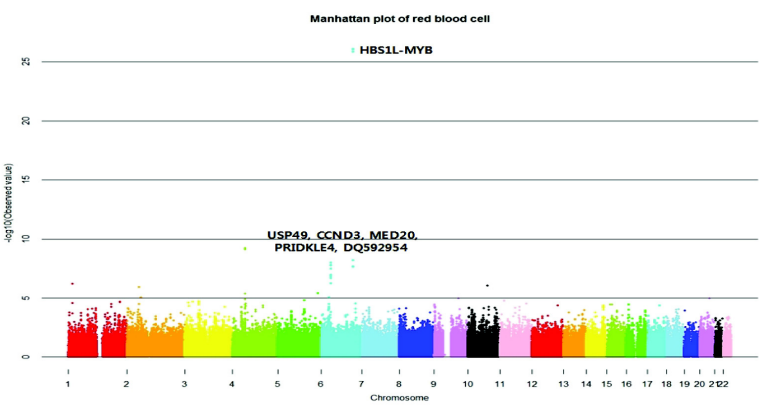

(c)

Fig. 1. Genome-wide association study of 8,842 Korean individuals for erythrocyte traits: (a) Hb, (b) Hct, and (c) RBC. Manhattan plots of log-transformed $p$-values with chromosome location. 


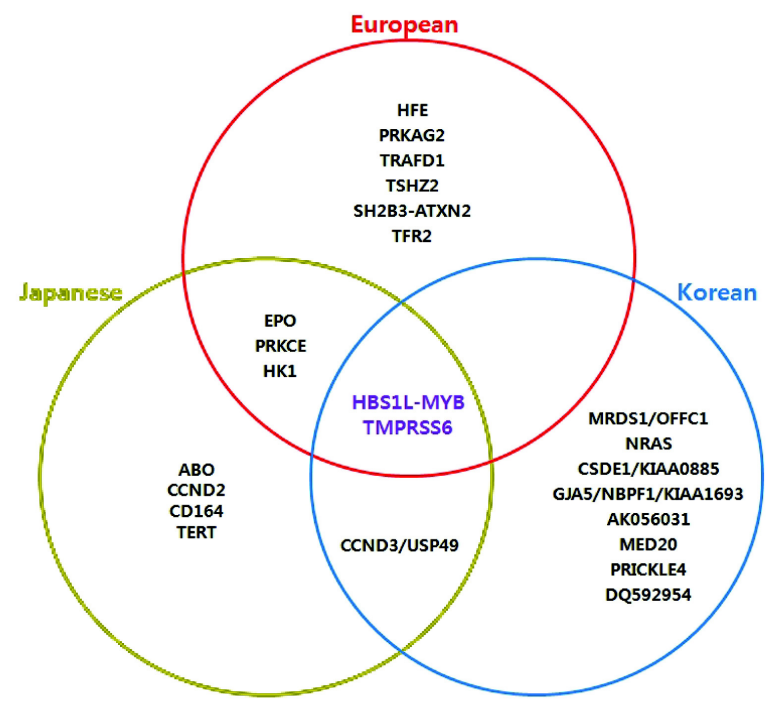

Fig. 2. Comparison of genes influencing erythrocyte traits among European, Japanese, and Korean. Two loci, HBS1L-MYB and TMPRSS6, were common among the three populations.

\section{Methods}

\section{Study samples and genotype data}

The samples and genotype data used in this study have been described by Cho et al. (2009). Briefly, through the Korea Association Resource (KARE) project that was initiated in 2007 10,038 participants were recruited from Ansan $(n=5,020)$ and Ansung $(n=5,018)$ population-based cohorts aged 40 to 69 . Among them 10,004 were genotyped using the Affymetrix Genome-Wide Human SNP array 5.0. After removing samples and markers that failed quality control test, a total of 352,228 markers in 8,842 individuals were used (Cho et al., 2009).

\section{SNP imputation}

SNP imputation has been described by Lee and Kim (2009). Briefly, using PLINK (Purcell et al., 2007) the KARE genotypes were supplemented by imputing SNP genotypes based on those of the unrelated Chinese in Beijing (CHB) and Japanese in Tokyo (JPT) panel of HapMap Phase II.

\section{Association analyses}

Erythrocyte traits were tested for association by linear regression analysis with an additive model after adjustment for age, sex and bmi as covariates using PLINK. The genome-wide level of significance threshold was set at $p<1 \times 10^{-6}$.

\section{Results}

In the current study, we performed GWAS for the three erythrocyte traits of 8,842 samples that are divided as 4,183 men and 4,659 women. $\mathrm{Hb}$, Hct and RBC of erythrocyte traits are commonly used in the diagnosis of anemia. Single marker association $p$-values were estimated from linear regression after adjusting age, sex and bmi, and then 40 loci were identified as strongly associated with erythrocyte traits having $p$ values lower than $1.00 \times 10^{-6}$ (Table 1, Fig. 1). These loci were mapped to $6 \mathrm{Hb}, 4 \mathrm{Hct}$ and $6 \mathrm{RBC}$ genes. We confirmed the previously reported associations of erythrocyte traits with the following four loci-: HBS1L-MYB, TMPRSS6, USP49 and CCND3 (Table 2, Fig. 3).

In the HBS1L-MYB intergenic region on chromosome 6q23, several SNPs showed genome-wide significant associations with $\mathrm{Hct}$ and $\mathrm{RBC}$. While the role of HBS1L is unknown, MYB has been associated with proliferation, survival, and differentiation of hematopoietic progenitor cells. In addition the intergenic region of the HBS1L gene and the MYB gene has been identified to be a quantitative trait locus controlling fetal hemoglobin level, and this region influences erythrocyte, platelet, and monocyte counts as well as erythrocyte volume and hemoglobin content. Like our findings, SNP rs4895441 is strongly associated with Hct and $\operatorname{RBC}\left(p=9.7 \times 10^{-10}\right.$, $\mathrm{p}=2.2 \times 10^{-15}$ ) in GWAS of European population. Other SNPs of HBS1L-MYB intergenic region were located in complete LD block with rs4895441 ( $\left.D^{\prime}=1.000, R^{2}=0.999\right)$. In the RBC, six SNPs of HBS1L-MYB intergenic region are as follows:rs11759553 $\left(p=8.33 \times 10^{-27}\right), \quad r s 4895440$ $\left(p=8.33 \times 10^{-27}\right), \quad r s 9389269 \quad\left(p=8.44 \times 10^{-27}\right), \quad r s 9402686$ $\left(p=8.44 \times 10^{-27}\right), \quad r s 4895441 \quad\left(p=1.44 \times 10^{-26}\right)$ and rs$9376092\left(p=1.46 \times 10^{-26}\right)$. Rs9402686, rs4895441 and rs9376092 were identified to associate with erythrocytes in previous studies of Europe, Japan and China.

TMPRSS6 encodes a type II transmembrane serine protease produced by the liver that regulates the expression of hepcidin (Finberg et al., 2008), which blocks iron absorption. And it is known that mutation in TMPRSS6 gene causes iron-refractory iron deficiency anemia (IRIDA) from other study. SNP rs5756505 in TMPRSS6 gene was associated with $\mathrm{Hb}\left(\mathrm{p}=4.57 \times 10^{-7}\right)$ and in complete LD with rs5756504 that was associated with $\mathrm{Hb}$ in GWAS of Japanese population $\left(\mathrm{R}^{2}=0.988\right)$ (Table 1).

CCND3 gene has roles in hematopoiesis. For example, Ccdn3 $3^{-1-}$ mice showed lethality due to heart abnormalities combined with severe anemia in other study (Katarzyna et al,. 2004). Two SNPs in CCND3 
Table 1. Genome-wide association studies for erythrocyte traits in 8,842 Korean individuals

\begin{tabular}{|c|c|c|c|c|c|c|c|c|}
\hline Trait & Chr & Gene & SNP & $\mathrm{bp}$ & Minor allele & MAF & Beta & $p$-value \\
\hline \multicolumn{9}{|c|}{ New associations } \\
\hline $\mathrm{Hb}$ & 1 & NRAS & Rs14804 & 115051366 & A & 0.01644 & -2.316 & $5.27 \times 10^{-9}$ \\
\hline $\mathrm{Hb}$ & 1 & CSDE1/KIAA0885 & Rs7555948 & 115089224 & $\mathrm{~T}$ & 0.01942 & -1.607 & $7.10 \times 10^{-7}$ \\
\hline $\mathrm{Hb}$ & 1 & GJA5/NBPF1 & Rs10793705 & 145706931 & $T$ & 0.4204 & -0.08616 & $7.72 \times 10^{-7}$ \\
\hline $\mathrm{Hb}$ & 6 & MRDS1/OFCC1 & Rs3765276 & 10090486 & G & 0.006144 & -3.332 & $2.73 \times 10^{-9}$ \\
\hline $\mathrm{Hb}$ & 6 & MRDS1/OFCC1 & Rs3765277 & 10090422 & G & 0.006146 & -3.332 & $2.74 \times 10^{-9}$ \\
\hline $\mathrm{Hb}$ & 6 & MRDS1/OFCC1 & Rs17621965 & 10105418 & $A$ & 0.01103 & -3.332 & $2.84 \times 10^{-9}$ \\
\hline $\mathrm{Hb}$ & 6 & MRDS1/OFCC1 & Rs 17543708 & 10069924 & $T$ & 0.01136 & -3.332 & $2.86 \times 10^{-9}$ \\
\hline $\mathrm{Hb}$ & 6 & MRDS1/OFCC1 & Rs10484262 & 10089479 & A & 0.01142 & -3.332 & $2.86 \times 10^{-9}$ \\
\hline $\mathrm{Hb}$ & 6 & MRDS1/OFCC1 & Rs 17544450 & 10097751 & A & 0.01142 & -3.332 & $2.86 \times 10^{-9}$ \\
\hline $\mathrm{Hb}$ & 6 & MRDS1/OFCC1 & Rs10484261 & 10091850 & $T$ & 0.01148 & -3.332 & $2.86 \times 10^{-9}$ \\
\hline $\mathrm{Hb}$ & 18 & AK056031 & Rs9676158 & 53555113 & $\mathrm{~T}$ & 0.09701 & -0.287 & $9.50 \times 10^{-7}$ \\
\hline Hct & 1 & NRAS & Rs14804 & 115051366 & A & 0.01644 & -6.696 & $7.33 \times 10^{-9}$ \\
\hline Hct & 1 & CSDE1/KIAA0885 & Rs7555948 & 115089224 & $T$ & 0.01942 & -4.638 & $9.54 \times 10^{-7}$ \\
\hline Hct & 6 & MRDS1/OFCC1 & Rs3765277 & 10090422 & G & 0.006146 & -8.157 & $6.16 \times 10^{-7}$ \\
\hline Hct & 6 & MRDS1/OFCC1 & Rs3765276 & 10090486 & G & 0.006144 & -8.158 & $6.16 \times 10^{-7}$ \\
\hline Hct & 6 & MRDS1/OFCC1 & Rs17621965 & 10105418 & $A$ & 0.01103 & -8.157 & $6.36 \times 10^{-7}$ \\
\hline Hct & 6 & MRDS1/OFCC1 & Rs17543708 & 10069924 & $T$ & 0.01136 & -8.156 & $6.38 \times 10^{-7}$ \\
\hline Hct & 6 & MRDS1/OFCC1 & Rs10484262 & 10089479 & $A$ & 0.01142 & -8.155 & $6.38 \times 10^{-7}$ \\
\hline Hct & 6 & MRDS1/OFCC1 & Rs10484261 & 10091850 & $T$ & 0.01148 & -8.155 & $6.38 \times 10^{-7}$ \\
\hline Hct & 6 & MRDS1/OFCC1 & Rs17544450 & 10097751 & $A$ & 0.01142 & -8.155 & $6.38 \times 10^{-7}$ \\
\hline $\mathrm{RBC}$ & 6 & USP49 & Rs33954419 & 41894114 & G & 0.4314 & 0.03213 & $9.91 \times 10^{-9}$ \\
\hline $\mathrm{RBC}$ & 6 & USP49 & Rs2488338 & 41916329 & G & 0.4309 & 0.03193 & $1.55 \times 10^{-8}$ \\
\hline $\mathrm{RBC}$ & 6 & USP49 & Rs2249703 & 41922738 & G & 0.4308 & 0.03193 & $1.55 \times 10^{-8}$ \\
\hline $\mathrm{RBC}$ & 6 & USP49 & Rs2253961 & 41934989 & G & 0.4309 & 0.03193 & $1.55 \times 10^{-8}$ \\
\hline $\mathrm{RBC}$ & 6 & USP49 & Rs2251084 & 41946097 & C & 0.4309 & 0.03193 & $1.55 \times 10^{-8}$ \\
\hline $\mathrm{RBC}$ & 6 & USP49 & Rs10498752 & 41876488 & $T$ & 0.4309 & 0.03193 & $1.55 \times 10^{-8}$ \\
\hline $\mathrm{RBC}$ & 6 & USP49 & Rs2185798 & 41881554 & G & 0.4309 & 0.03193 & $1.55 \times 10^{-8}$ \\
\hline $\mathrm{RBC}$ & 6 & USP49 & Rs7753507 & 41892072 & c & 0.4309 & 0.03193 & $1.55 \times 10^{-8}$ \\
\hline $\mathrm{RBC}$ & 6 & USP49 & Rs2025951 & 41935171 & G & 0.4313 & 0.03216 & $9.87 \times 10^{-9}$ \\
\hline $\mathrm{RBC}$ & 6 & USP49 & Rs2254474 & 41951022 & G & 0.4309 & 0.03193 & $1.55 \times 10^{-8}$ \\
\hline $\mathrm{RBC}$ & 6 & USP49 & Rs2254805 & 41954088 & G & 0.4309 & 0.03193 & $1.55 \times 10^{-8}$ \\
\hline RBC & 6 & USP49 & Rs9381096 & 41929115 & $A$ & 0.2128 & 0.05017 & $2.02 \times 10^{-7}$ \\
\hline $\mathrm{RBC}$ & 6 & USP49 & Rs1887718 & 41965669 & $T$ & 0.4309 & 0.03193 & $1.55 \times 10^{-8}$ \\
\hline RBC & 6 & CCND3 & Rs4623235 & 42032831 & A & 0.4409 & 0.0324 & $1.70 \times 10^{-8}$ \\
\hline RBC & 6 & CCND3 & Rs11970772 & 42033268 & A & 0.4894 & -0.03015 & $3.47 \times 10^{-8}$ \\
\hline $\mathrm{RBC}$ & 6 & MED20 & Rs2274578 & 41996805 & G & 0.4309 & 0.03193 & $1.55 \times 10^{-8}$ \\
\hline RBC & 6 & PRICKLE4 & Rs6905726 & 41858790 & $\mathrm{~T}$ & 0.4309 & 0.03193 & $1.55 \times 10^{-8}$ \\
\hline RBC & 6 & PRICKLE4 & Rs6925777 & 41858800 & C & 0.4309 & 0.03193 & $1.55 \times 10^{-8}$ \\
\hline RBC & 6 & DQ592954 & Rs8393 & 41865814 & C & 0.4309 & 0.03193 & $1.55 \times 10^{-8}$ \\
\hline \multicolumn{9}{|c|}{ Replication of previous reports } \\
\hline $\mathrm{Hb}$ & 22 & TMPRSS6 & Rs5756505 (Kamatani et al.) & 35797300 & C & 0.4987 & 0.08523 & $4.57 \times 10^{-7}$ \\
\hline Hct & 6 & HBS1L-MYB & Rs9389269 (Ganesh et al.) & 135468852 & C & 0.3541 & -0.2985 & $1.12 \times 10^{-7}$ \\
\hline Hct & 6 & HBS1L-MYB & Rs9402686 (Ganesh et al.) & 135469510 & $A$ & 0.3542 & -0.2985 & $1.12 \times 10^{-7}$ \\
\hline Hct & 6 & HBS1L-MYB & Rs11759553 (Ganesh et al.) & 135463989 & $\mathrm{~T}$ & 0.354 & -0.2985 & $1.12 \times 10^{-7}$ \\
\hline Hct & 6 & HBS1L-MYB & Rs4895440 (Ganesh et al.) & 135468251 & $\mathrm{~T}$ & 0.3541 & -0.2985 & $1.12 \times 10^{-7}$ \\
\hline Hct & 6 & HBS1L-MYB & Rs9376092 (Ganesh et al.) & 135468837 & A & 0.3538 & -0.2958 & $1.45 \times 10^{-7}$ \\
\hline Hct & 6 & HBS1L-MYB & Rs4895441 (Ganesh et al.) & 135468266 & $\mathrm{G}$ & 0.3538 & -0.2957 & $1.46 \times 10^{-7}$ \\
\hline $\mathrm{RBC}$ & 6 & USP49 & Rs6899876 (Kamatani et al.) & 41904948 & c & 0.2329 & 0.04638 & $1.40 \times 10^{-7}$ \\
\hline $\mathrm{RBC}$ & 6 & USP49 & Rs9381097 (Kamatani et al.) & 41955778 & $A$ & 0.2327 & 0.04683 & $1.09 \times 10^{-7}$ \\
\hline RBC & 6 & MED20 & Rs3806113 (Kamatani et al.) & 41984313 & $T$ & 0.2357 & 0.04418 & $5.81 \times 10^{-7}$ \\
\hline $\mathrm{RBC}$ & 6 & HBS1L-MYB & Rs11759553 (Ganesh et al.) & 135463989 & $\mathrm{~T}$ & 0.354 & -0.06687 & $8.33 \times 10^{-27}$ \\
\hline $\mathrm{RBC}$ & 6 & HBS1L-MYB & Rs4895440 (Ganesh et al.) & 135468251 & $\mathrm{~T}$ & 0.3541 & -0.06687 & $8.33 \times 10^{-27}$ \\
\hline $\mathrm{RBC}$ & 6 & HBS1L-MYB & Rs9389269 (Ganesh et al.) & 135468852 & C & 0.3541 & -0.06686 & $8.44 \times 10^{-27}$ \\
\hline RBC & 6 & HBS1L-MYB & Rs9402686 (Ganesh et al.) & 135469510 & $A$ & 0.3542 & -0.06686 & $8.44 \times 10^{-27}$ \\
\hline RBC & 6 & HBS1L-MYB & Rs4895441 (Ganesh et al.) & 135468266 & $G$ & 0.3538 & -0.06653 & $1.44 \times 10^{-26}$ \\
\hline $\mathrm{RBC}$ & 6 & HBS1L-MYB & Rs9376092 (Ganesh et al.) & 135468837 & $A$ & 0.3538 & -0.06652 & $1.46 \times 10^{-26}$ \\
\hline
\end{tabular}




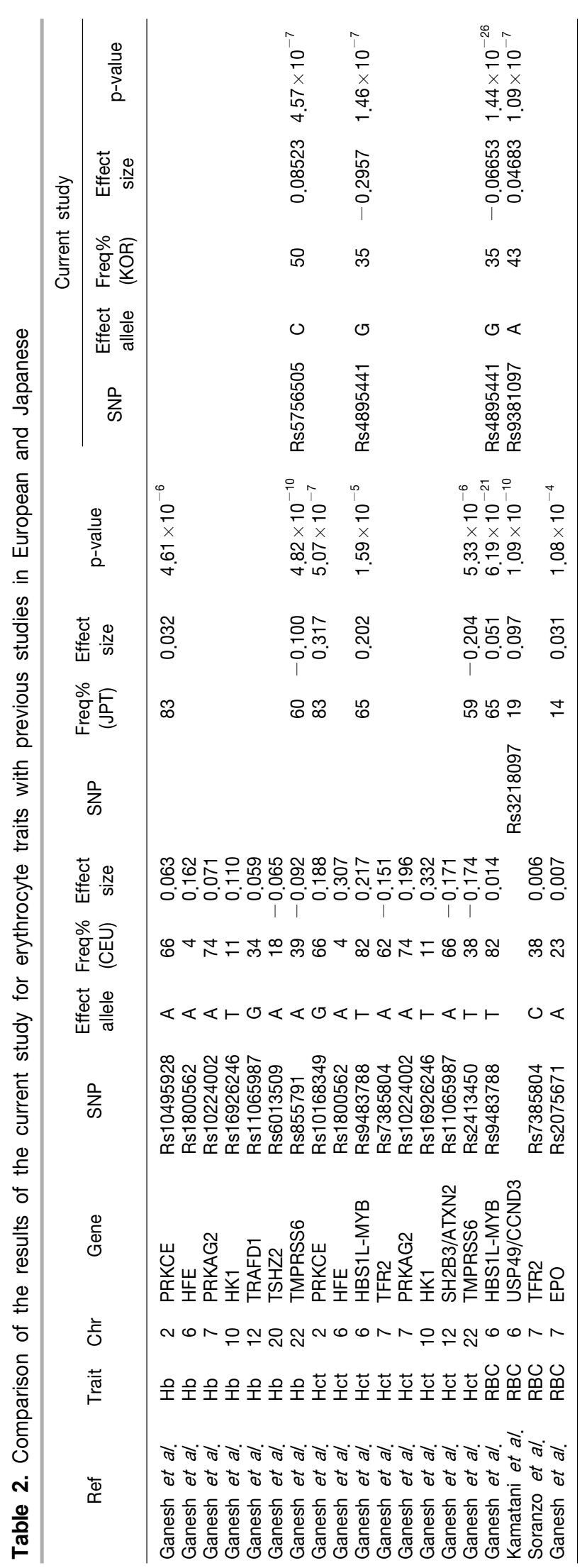

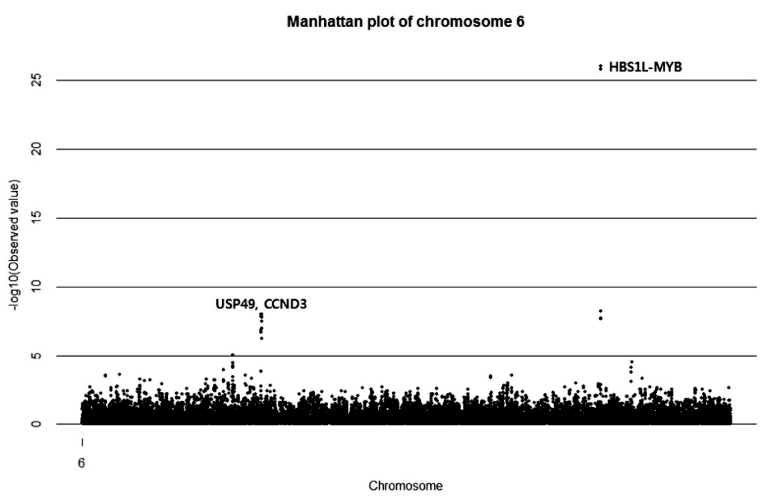

Fig. 3. Significant associations on chromosome 6 in RBC. HBS1L-MYB, CCND3 and USP49 loci were also previously reported in other studies.

gene on chromosome 6p21.1 were associated with RBC (rs4623235, $\mathrm{p}=1.70 \times 10^{-8}$ and $\mathrm{rs} 11970772$, $\left.\mathrm{p}=3.47 \times 10^{-8}\right) ; \quad$ rs11970772 was significantly associated with mean corpuscular volume (MCV) of erythrocyte traits in previous study.

USP49 gene is located so close to the CCND3 gene. Two SNPs of USP49 found associated with RBC in our study were in high LD with SNP rs3218097 of CCND3 reported in Japanese GWAS (rs9381097 $D^{\prime}=0.952 R^{2}=$ 0.893 , rs6899876 $\left.D^{\prime}=0.952 R^{2}=0.893\right)$. SNP rs3806113 of MED20 gene was also in high LD with SNP 3218097 $\left(D^{\prime}=0.972, R^{2}=0.851\right)$ (Table 1, 2).

We found several loci associated with erythrocyte traits that have not been reported previously as follows: MRDS1/OFCC1, NRAS, CSDE1, GJA5/NBPF1, MED20, PRICKLE4, DQ592954, and AK056031 (Table 1). Most of these loci were located on chromosome 6. For example, newly found genes such as MED 20, PRICKLE4 and DQ592954 as well as previously reported USP49 and CCND3 are located on chromosome 6p21.1. While their roles in erythrocyte traits are largely unknown, we presume that these loci affect RBC.

\section{Discussion}

In the current study, we identified 40 associations for three erythrocyte traits at genome-wide significance levels $\left(p<1 \times 10^{-6}\right)$ in 8,842 Korean. Of these loci, ten SNPs were previously reported, while 30 were novel, and their neighboring genes were known to be involved with iron homeostasis, erythropoiesis, and globin synthesis. Measurement of $\mathrm{RBC}, \mathrm{Hb}$ and Hct, which are mainly quantitative measures of hemoglobin per erythrocytes, are parameters for the detection of blood disease associated erythrocyte in clinic. Across the three erythrocyte traits studied, the strongest signal was 
found in the HBS1L-MYB locus on chromosome 6q23 (Table 1). Although its association with $\mathrm{Hct}$ and RBC only is listed in Table 1, its SNPs also have associations with $\mathrm{Hb}\left(\mathrm{r} s 11759553 \mathrm{p}=8.07 \times 10^{-6}\right.$, rs $4895440 \mathrm{p}=8.07 \times$ $10^{-6}, r s 9402686 p=8.41 \times 10^{-6}, r s 9389269 p=8.41 \times 10^{-6}$, rs4895441 $\mathrm{p}=9.82 \times 10^{-6}, \quad$ rs9376092 $\mathrm{p}=1.02 \times 10^{-5}$ ). CCND3 encodes cyclin D3, which has been known to be critical for the expansion of hematopoietic stem cells, as mice lacking D-cyclins develop severe anemia in experiment ${ }^{7}$. Hence it is likely that genetic factors in cyclin-related genes influence erythrocyte traits. Comparisons of erythrocyte traits among European, Japanese and Korean, confirm that HBS1L-MYB and TMPRSS6 loci are strongly associated with erythrocyte traits both in European and Asian. On the other hand, CCND3 and USP49 genes influencing RBC are identified in only Asian. Besides, many other genes belonged exclusively to each population (Fig. 2).

The novel loci reported in the current study need further study to confirm whether they have roles in biological processes associated erythrocytes.

\section{Acknowledgements}

The KARE genotype and epidemiological data were gratefully made available by National Institute of Health, Korea Center for Disease Control, which supported this work through the KARE Analysis Consortium. We would like to thank the help and support of all the staff at $\mathrm{KNIH}$.

\section{References}

Cho, Y.S., Go, M.J., Kim, Y.J., Heo, J.Y., Oh, J.H., Ban, H.J., Yoon, D., Lee, M.H., Kim, D.J., Park, M., Cha, S.H., Kim, J.W., Han, B.G., Min, H., Ahn, Y., Park, M.S., Han, H.R., Jang, H.Y., Cho, E.Y., Lee, J.E., Cho, N.H., Shin, C., Park, T., Park, J.W., Lee, J.K., Cardon, L., Clarke, G., McCarthy, M.I., Lee, J.Y., Lee, J.K., Oh, B., and Kim, H.L. (2009). A large-scale genome-wide association study of Asian populations uncovers genetic factors influencing eight quantitative traits. Nat. Genet. 41, 527-534.

Finberg, K.E., Heeney, M.M., Campagna, D.R., Aydinok, Y., Pearson, H.A., Hartman, K.R., Mayo, M.M., Samuel, S.M., Strouse, J.J., Markianos, K., Andrews, N.C., and Fleming, M.D. (2008). Mutations in TMPRSS6 cause iron-refractory iron deficiency anemia (IRIDA). Nat. Genet. 40, 569-571.

Ganesh, S.K., Zakai, N.A., van-Rooij, F.J., Soranzo, N., Smith, A.V., Nalls, M.A., Chen, M.H., Kottgen, A., Glazer, N.L., Dehghan, A., Kuhnel, B., Aspelund, T., Yang, Q., Tanaka, T., Jaffe, A., Bis, J.C., Verwoert, G.C., Teumer, A., Fox, C.S., Guralnik, J.M., Ehret, G.B., Rice, K., Felix, J.F., Rendon, A., Eiriksdottir, G., Levy, D., Patel, K.V., Boerwinkle, E., Rotter, J.I., Hofman, A., Sambrook, J.G., Hernandez, D.G., Zheng, G., Bandinelli, S., Singleton,
A.B., Coresh, J., Lumley, T., Uitterlinden, A.G., Vangils, J.M., Launer, L.J., Cupples, L.A., Oostra, B.A., Zwaginga, J.J., Ouwehand, W.H., Thein, S.L., Meisinger, C., Deloukas, P., Nauck, M., Spector, T.D., Gieger, C., Gudnason, V., van Duijn, C.M., Psaty, B.M., Ferrucci, L., Chakravarti, A., Greinacher, A., O'Donnell, C.J., Witteman, J.C., Furth, S., Cushman, M., Harris, T.B., and Lin, J.P. (2009). Multiple loci influence erythrocyte phenotypes in the CHARGE Consortium. Nat. Genet. 41, 1191-1198.

Katarzyna, K., Maria, A., Ciemerych, V., I. R., Hirokazu, S., Agnieszka, Z., Ewa, S., Yan, G., Qunyan, Y., Shoumo, B., Roderick, T.B., Koichi, A., and Piotr, S. (2004). Mouse Development and Cell Proliferation in the Absence of D-Cyclins. Cell 118, 477-491.

Lee, K., and Kim, S. (2009). A scheme for filtering SNPs imputed in 8,842 Korean individuals based on the international HapMap project data. Genomics Inform. 7, 136-140

Nuinoon, M., Makarasara, W., Mushiroda, T., Setianingsih, I., Wahidiyat, P.A., Sripichai, O., Kumasaka, N., Takahashi, A., Svasti, S., Munkongdee, T., Mahasirimongkol, S., Peerapittayamongkol, C., Viprakasit, V., Kamatani, N., Winicha-

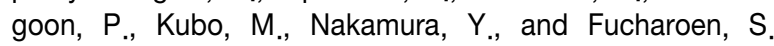
(2009). A genome-wide association identified the common genetic variants influence disease severity in beta(0)-thalassemia/hemoglobin E. Hum. Genet. 127, 303-314.

Purcell, S., Neale, B., Todd-Brown, K., Thomas, L., Ferreira, M.A., Bender, D., Maller, J., Sklar, P., de Bakker, P.I., Daly, M.J., and Sham, P.C. (2007). PLINK: a tool set for whole-genome association and population-based linkage analyses. Am. J. Hum. Genet. 81, 559-575.

Soranzo, N., Spector, T.D., Mangino, M., Kühnel, B., Rendon, A., Teumer, A., Willenborg, C., Wright, B., Chen, L., Li, M., Salo, P., Voight, B.F., Burns, P., Laskowski, R.A., Xue, Y., Menzel, S., Altshuler, D., Bradley, J.R., Bumpstead, S., Burnett, M.S., Devaney, J., Döring, A., Elosua, R., Epstein, S.E., Erber, W., Falchi, M., Garner, S.F., Ghori, M.J., Goodall, A.H., Gwilliam, R., Hakonarson, H.H., Hall, A.S., Hammond, N., Hengstenberg, C., Illig, T., König, I.R., Knouff, C.W., McPherson, R., Melander, O., Mooser, V., Nauck, M., Nieminen, M.S., O'Donnell, C.J., Peltonen, L., Potter, S.C., Prokisch, H., Rader, D.J., Rice, C.M., Roberts, R., Salomaa, V., Sambrook, J., Schreiber, S., Schunkert, H., Schwartz, S.M., Serbanovic-Canic, J., Sinisalo, J., Siscovick, D.S., Stark, K., Surakka, I., Stephens, J., Thompson, J.R., Völker, U., Völzke, H., Watkins, N.A., Wells, G.A., Wichmann, H.E., Van-Heel, D.A., Tyler-Smith, C., Thein, S.L., Kathiresan, S., Perola, M., Reilly, M.P., Stewart, A.F., Erdmann, J., Samani, N.J., Meisinger, C., Greinacher, A., Deloukas, P., Ouwehand, W.H., and Gieger, C. (2009). A genome-wide meta-analysis identifies 22 loci associated with eight hematological parameters in the HaemGen consortium. Nat. Genet. 41, 1182-1190.

Yoichiro, K., Koichi, M., Yukinori, O., Michiaki, K., Naoya,

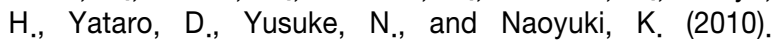
Genome-wide association study of hematological and biochemical traits in a Japanese population. Nat. Genet. 42, 210-215. 